matical study (pp. 10-16) by Dr. Shrubsall of the statistics of growth. The general results might with advantage have been further elaborated on the practical side. As to teeth, there is a strong plea for school dental clinics on the model of Strassburg.

A special investigation as to tuberculosis of the lungs in school children was undertaken by Dr. Squire and Dr. Annie Gowdey. Of actual phthisis, only 335 cases (i.e. 0.55 per cent.) were found among 58,934 children. The sections on hearing and acuity of vision contain much fresh material. One of the most important sections deals with the "development of articulatory capacity for consonantal sounds" (p. 27). Considerable detail is given of the methods of testing, and 105,000 tests were made on some 3000 children. The results are given in an exact quantitative way, capable of analytical study. This department is of immense importance to the teacher, as the work already done in phonetics has abundantly shown. As to fatigue, some new curves from rifle-shooting are given. It is found that the curve improves with a little practice, coordination improving very rapidly. Cigarette-smoking was found to impair the capacity to shoot straight.

There are the usual sections dealing with the inspection of defective children and cripples, country homes, infectiou: diseases, adenoids, \&c. ; but two sections must be specially named, one on the artificial lighting of school-rooms and the other on the mental and physical effects of bad ventilation. In both researches the practical results are very definite, and ought to be driven home among teachers and architects alike. Of the ventilation research, some provisional conclusions are:- "Temperatures above $65^{\circ} \mathrm{F}$. give rise to definite subjective symptoms, slackness and inattention in some, headaches in others. Although it is not easy to assert definite mental alteration till about $70^{\circ}$ F." "Symptoms do not appear at $65^{\circ}$ if the air is kept in gentle movement by a fan in the room. With temperatures $70^{\circ} \mathrm{F}$. and above, other factors being normal, there are marked symptoms and very evident deterioration in mental alertness and accuracy." At low temperatures. relative humidity does not affect the mental capacity of children, but increase of humidity increases the effects of high temperatures. Carbonic acid gas in considerable excess increases markedly the fatigue of the children. Exact details are given of the methods used.

The London County Council is to be congratulated on the issue of this mass of original and important observations in so many departments of medical inspection. Dr. Kerr's reports show the great educational possibilities of the system, which, under his guidance, has revealed many new regions for clinical and scientific research.

\section{THFORY OF THE MIRAGE.}

THE theory of the mirage forms the subject of several recent papers by Prof. Antonio Garbasso. In notes contributed to the Atti dei Lincei, xvi. (2), I, 8, the author discusses the propagation of light in a heterogeneous medium, making use of the principle of least time. and considering the case of space of any number of dimensions defined by curvilinear coordinates. The space in question is supposed to be subject to the usual assumption that the square of the line-element is a homogeneous quadratic function of the differentials of the coordinates. As might be expected from the principle of least action (an analogy the applications of which to the problem are probably already known), the equations of the path can be reduced to the form of the ordinary equations of dynamics by a suitable choice of the characteristic function. The applications to the mirage itself are discussed in a paper in the Memorie of the Turin Academy, 1907. Prof. Garbasso claims that while the phenomenon has been studied both experimentally and theoretically, his present work fills a sap in the literature by establishing agreement of a quantitative character between the results of calculation and those of experiment.

Two kinds of mirage are distinguished, one due to the variations of density caused by diffusion between two fluids of different refrangibility initially having a plane of separation; this is called the mirage of Vince. The second kind, called the mirage of Monge, depends on diffusion outwards from a plane boundary maintained in definitely at the same conditions. The former condition gives three images, two direct and one inverted; the latter gives only the reflected image. Prof. Garbasso calculates the law of density from the equations of diffusion, and thus determines the equations of the trajectories of the rays of light and the form of the wave-front.

The final comparison with experiment is discussed in a paper by Luigi Rolla, also in the Memorie of the Turin Academy. In it the last-named author describes experiments showing how, not only has Wollaston's original artificial mirage of the Vince type been reproduced with its three images, but also the Monge mirage has been imitated, and in both cases the trajectories of the rays have been detcrmined by observation and compared with results of theory. Moreover, a mirage with five images, observed by Parnell at Folkestone in 1869 , was realised by placing over a layer of carbon bisulphide a mixture of equal parts by volume of alcohol and chloroform. Owing to the unequal rates of diffusion, the conditions give rise to five images, and this and other experiments are shown to be suitable for lecture-room demonstration.

By taking a block of gelatin containing a cavity filled with liquid which gradually diffuses into the gelatin, the corresponding images for a cylindrical or spherical distribution of density have been also produced and compared with the results of mathematical calculation.

The first and second figures show the mirages of a diaphragm somewhat in the shape of a ship produced by the medium formed by diffusion between alcohol an.1

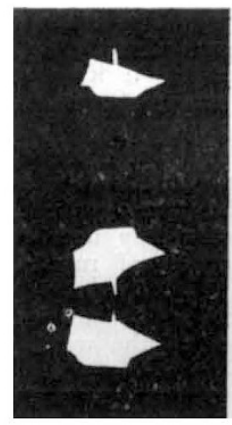

FIG. I.

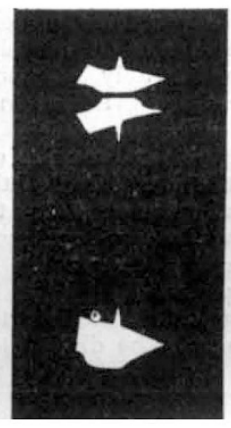

FIG. 2.

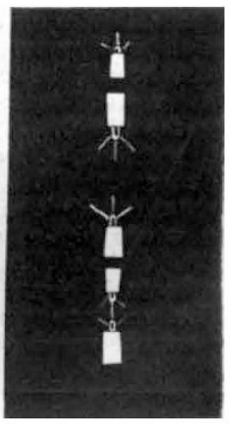

FIG. 3. bisulphide of carbon. Fig. I represents the appearance after a few hours, Fig. 2 after several days. Fig. 3 shows the five images obtained by diffusion between bisulphide of carbon and a mixture of alcohol and chloroform.

G. H. B.

\section{A CONTRIBUTION TO THE HISTORY OF IRONCLADS.}

I ORD ROSSE has made an interesting contribution to the history of ironclads by placing at the service of the Institution of Naval Architects copies of letters written by his father to various distinguished men in the years 1854-5. From these letters it appears that the late Lord Rosse not merely appreciated the importance of armour protection against horizontal shell fire, but satisfied himself that it was possible by means of suitable proportions to secure ample stability in ironclad ships. Naval officers were then disposed to think that the "top-weight" inevitable with heavy loads of armour would make vessels unstable. Lord Rosse proposed the construction of ironclad floating batteries of moderate size; they were intended to fight in smooth water, and consequently were to carry their guns at a small height above water. The exposed sides were to be armoured with 5 inches of iron, and the upper decks to be covered with 2 -inch plating.

In a letter to Sir John Burgoyne dated June 26, 1854 , Lord Rosse proposed an armament of sixteen heavy guns; the draught of water was not to exceed 12 feet to 13 feet, and the vessel was estimated to be about isoo tons. He 